\title{
Performance of a Non-contact Handling Device using Swirling Flow with Various Gap Height
}

\author{
Shouichiro Iio*1, Masako Umebachi ${ }^{* 2}$, Xin $\mathrm{Li}^{* 3}$, \\ Toshiharu Kagawa*3, Toshihiko Ikeda*1 \\ *1 Department of Environmental Science and Technology, Shinshu University, 4- \\ 17-1 Wakasato, Nagano, 380-8553, Japan. \\ +81-26-269-5111, +81-26-269-5130, E-mail: shouiio@shinshu-u.ac.jp \\ *2 Department of Environmental Science and Technology, Graduate school of \\ Shinshu University, 4-17-1 Wakasato, Nagano, 380-8553, Japan.
}

*3 Precision and Intelligence Laboratory, Tokyo Institute of Technology, R245,4259 Nagatsuta-chou, Midori-Ku, Yokohama, 226-8503, Japan.

ABSTRACT: Vortex levitation can achieve non-contact handling by blowing air into a vortex cup through a tangential nozzle to generate a swirling flow. In this paper, we focused on the relationship between the sucking pressure and the flow dynamics when gap distance from the cup to a work piece changes. Then simultaneous measurement of a pressure and a flow field in the cup was performed. As a result, the mean pressure changes and the pressure fluctuation inside the cup enhances with increasing the gap height. Especially, periodic pressure perturbation is observed with wide gap height and it synchronizes with the eccentric rotation of the swirling flow. It is also found that the rotation axis of swirling flow steadily-inclines against the central axis of the cup for appropriate gap height.

Keywords: Non-contact Handling, Swirling Flow, Gap Height, Simultaneous Measurement, Pressure Measurement, PTV

\section{Introduction}

In semiconductor manufacturing, wafers or glass substrates are conveyed in contact with a handling device for suctioning. The direct contact between the work and the handling device is often accompanied by surface scratching, static electricity and particle contamination. Non-contact and stable conveyance methods are desired to improve production quality and efficiency. As wafers and glass substrates get thinner and larger, the requirements of the non-contact handling of works become higher. In order to avoid contact between handling devices and work pieces, many non-contact handling approaches have been proposed and have proven effective. These methods typically employ magnetic, electrostatic and pneumatic levitation (Brandt 1989; Vandaele et al. 2005). Both magnetic and electric levitation are restricted to conductive materials and the lifting force depends on material properties. Pneumatic levitation approaches use air flow to apply a sucking force to a work piece. Because air flow is magnetic free and generates little heat, pneumatic approaches can be applied any material: insulator or conductor, magnetic or nonmagnetic, rigid or non-rigid (Davis et al. 2008; Vandaele et al. 2005). Furthermore, pneumatic approaches require no control loop to obtain a stable state and simple structures are nearly maintenance free. One typical pneumatic approach based upon Bernoulli principle that is most often used in practical applications is called Bernoulli levitation (Davis et al. 2008; Dini et al. 2009; Waltham et al. 2003). Recently, Dini et al.(2009) investigated the characteristics of different gripper configurations and clarified that the use of a deflector having a small angle and radial venturi channels on the plate give positive effects to the grasping force. However, Bernoulli levitation needs large air consumption often leads to a great air power loss through supply pipes (Davis et al. 2008). This paper focuses on a new pneumatic non-contacting handling approach named vortex levitation that uses swirling flow. Similar to cyclones where low pressure is caused by air swirling, a simple structure called the vortex cup is used. As can be seen in Fig. 1, the cup is composed of a circular cylinder and a tangential nozzle. A working fluid is issued from the nozzle, 
and then spins along the circular wall to create a negative pressure in the central area. This negative pressure will be applied as a sucking force to a work piece placed under the cup. Because the working fluid is supplied continuously, the work piece will keep levitating with a gap from the cup. For this reason, the work piece will be held at an equilibrium position where the weight of work piece is balanced with the sucking force. Many previous studies reported nature of swirling flows in cylindrical chambers and circular pipes (Ito et al. 1979; Kitoh 1991; Nissan et al. 1961), and its technical applications include separation of particles by cyclones and improvement of combustion by swirl burners, among others (Cortes et al. 2007; Kumar et al. 1993; Nishimura et al. 1990). But these previous results can not be applied directly to the handling device because it has an opening gap at one side of circular cylinder.

$\mathrm{Li}$ et al. $(2007,2008)$ reported the relationship between sucking force and gap distance. Iio et al. (2008) showed the velocity distribution in a vortex cup and revealed that the swirl rotation axis inclines against the center of the cup. The previous studies on the vortex cup clarified the relationships between the sucking force and the gap height, and between the swirling flow behavior and the gap height. It is, however, still unclear the direct relationship between the sucking force and swirling flow behavior. It is naturally thought that the swirling flow motion will have a close relationship with the sucking force. In this paper, simultaneous measurements of pressure and flow fields were performed to clarify the direct relationship between the sucking force and the swirling flow at various gap heights. Figure 2 is an image of a manipulator for practical applications which is equipped with vortex cups to achieve better stability and a larger sucking force. In this paper, only one typical sized vortex cup is used for experiments.

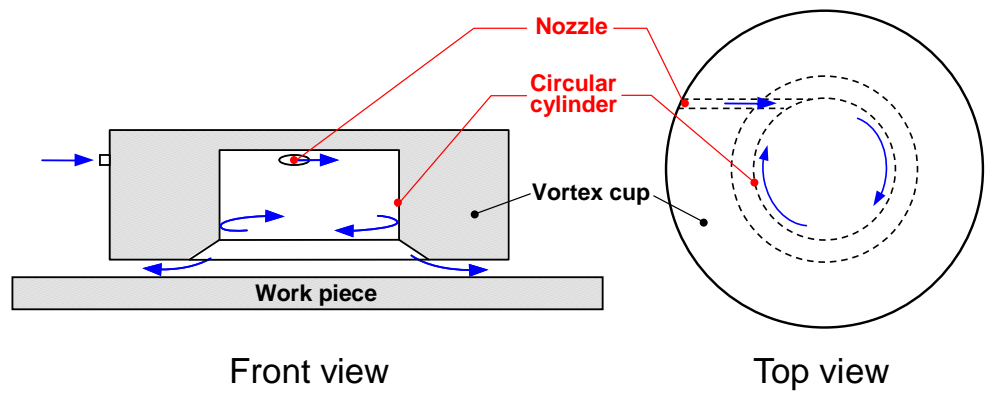

Fig. 1 Mechanism of vortex cup.

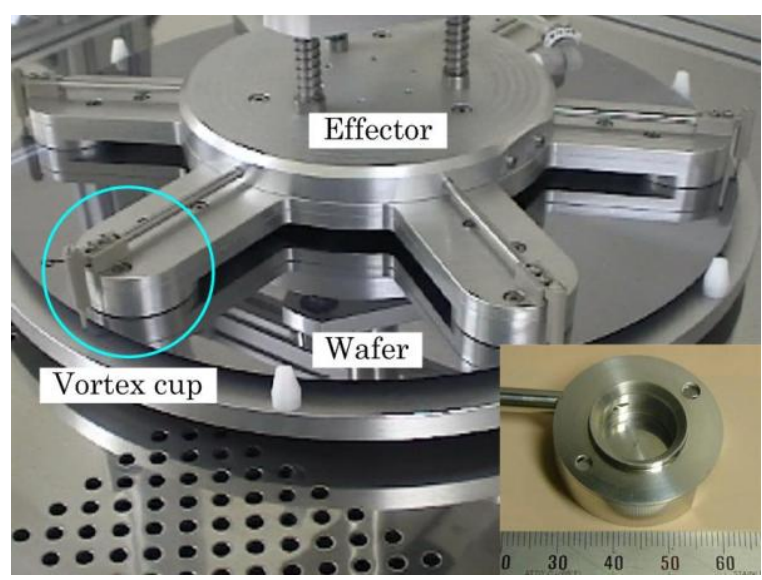

Fig. 2 Non-contact handling system by using swirling flow

\section{Experimental apparatus}

The arrangement of the experimental setup is shown in Fig. 3. In this experiment, water was used as a working fluid to visualize easily. For the air-flow cup, Li et al. reported that the swirl velocity is dominant in the cup, and its velocity reaches at approximately $50 \mathrm{~m} / \mathrm{s}$. In this case, the air compressibility is negligible. Water in a head tank with an overflow system goes through the water-supplying pipe, and then is adjusted to appropriate flow rate by hand valves, and enters the vortex cup placed in a test section from the nozzle. The issued water generates a swirling flow in the cup, and then goes out the test section through the gap between the cup and the work piece. The overflow system enables stable water supplying. The cup was fixed at the bottom of the test section such that the open-end of the cup is in an upward direction. 
The detail configuration of the cup is shown in Fig. 4. Enlarged cup model was made in order to keep a flow similarity for low swirl velocity. The both of the cup model and the work piece were made of transparent acrylic resin for easy visualized access into the cup. The cup was similar shape to the practical cup shown in Fig. 2, the inner diameter of the cup, $D$, was $72 \mathrm{~mm}$, the height of circular cylinder in the cup, $H$, was $44 \mathrm{~mm}$, and the aspect ratio of the cup, $H / D$, was 0.61 . The inner edge of the cup was chamfered by $10 \mathrm{~mm}$ at an angle $45^{\circ}$. The inner diameter of the nozzle, $d$, was $3.2 \mathrm{~mm}$, the nozzle height from the bottom was set at $4 \mathrm{~mm}$. The work piece was fixed on the cup at the setting gap height $\delta$. The rotating coordinate system was defined such as shown in Fig. 4. The origin was fixed at the cup center on the nozzle height, the $z$-axis was in the depth direction, and the $r$-axis was in the radius direction. An angle in the circumference direction was defined as $\theta$. A mean jet velocity at the cross section of the nozzle exit was defined as $V_{i n}$, and the outer circumference flow velocity was defined as $V_{w}$. The air-flow cup is used under the Reynolds number of $R e=58000$ based on the air flow velocity near the inner wall by Li et al.(2007). In this study, $V_{\text {in }}$ was set at $3.4 \mathrm{~m} / \mathrm{s}$ to establish the flow similarity between the air flow cup and the enlarged cup using water flow. In this case, $V_{w}$ was $0.81 \mathrm{~m} / \mathrm{s}$, the flow rate through the nozzle was set at $Q=27.3 \times 10^{-6} \mathrm{~m}^{3} / \mathrm{s}$.

The photographs to be shown here were captured via high speed digital video camera (DITECT, K- II , 640×480 pixel, $200 \mathrm{fps}$ ). The light sheet source was used 800 watt halogen bulb, and was illuminated at specific cross section. A nylon 12 powder (mean diameter $150 \mu \mathrm{m}$, specific gravity 1.02) was used as a tracer particle, and was seeded far upstream of the nozzle. The dynamic pressure inside the cup was measured with a sampling frequency of $1 \mathrm{kHz}$ on the cup wall or at the center of the work piece via Validyne DP-15 strain-gauge transducer. The pressure defined as $p$ means the differential pressure between the pressure tap holed at the above mentioned positions and the outside of the cup.

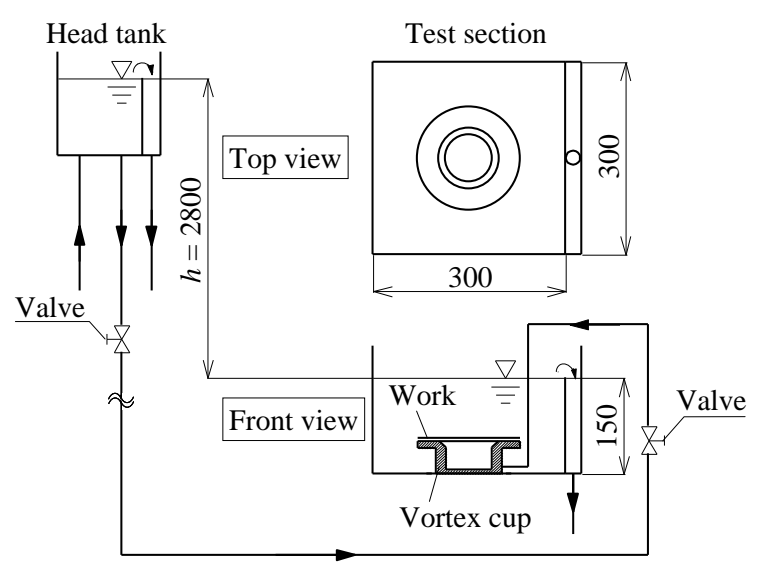

Fig. 3 Experimental setup.

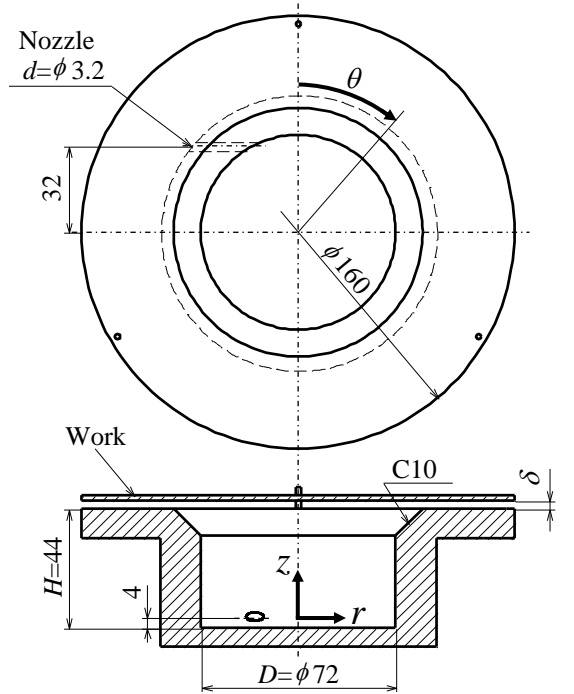

Fig. 4 Configuration of vortex cup

\section{Experimental results and discussion}

\subsection{Pressure measurement}

Figure 5 shows the mean sucking pressure, $p_{a v e}$, at various gap heights. The pressure tap is placed at the center of the work piece. The pressure indicates positive value for the smallest gap height, drastically decreases with increasing the gap height, and reaches the minimum at $\delta / H$ $=0.027$, and then gradually increases with the gap height. This result well agrees with that of the past studies (Li et al. 2007, 2008). For air-flow cup used practically, the gap height changes with the weight of work piece or with the supplying air flow rate. Next, Fig. 6 illustrates the sucking pressure fluctuations with different gap height, and Fig. 7 shows the results of spectrum analysis of the pressure fluctuations shown in Fig. 6. The spectrum result was extracted the frequency which was lower than $10 \mathrm{~Hz}$. As can be seen from these graphs, it is clearly observed that the amplitude of pressure fluctuation increases with the gap height. The dominant frequency is recognized for $\delta / H \geq 0.036$. In particular, the spectrum intensity of dominant frequency increases and the 
dominant frequency shifts lower side when the gap height increases for $\delta / H \geq 0.041$. But the dominant frequency is around $1 \mathrm{~Hz}$. It is found that the periodical phenomenon occurred in the vortex cup under the wide gap condition.

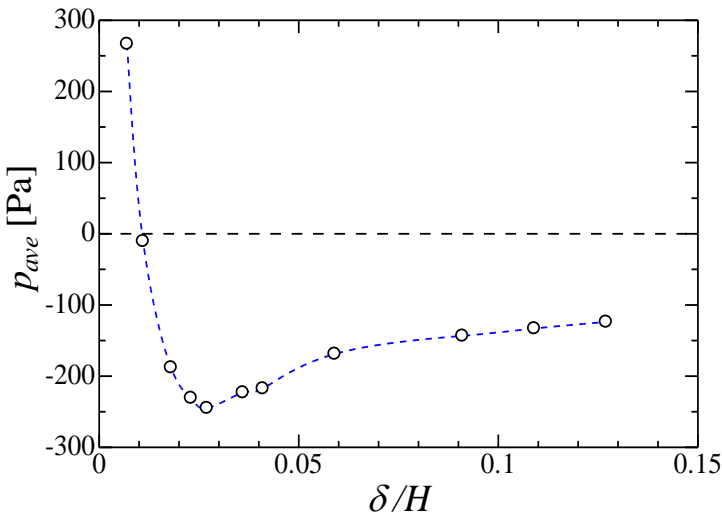

Fig. 5 Sucking pressure at various gap height

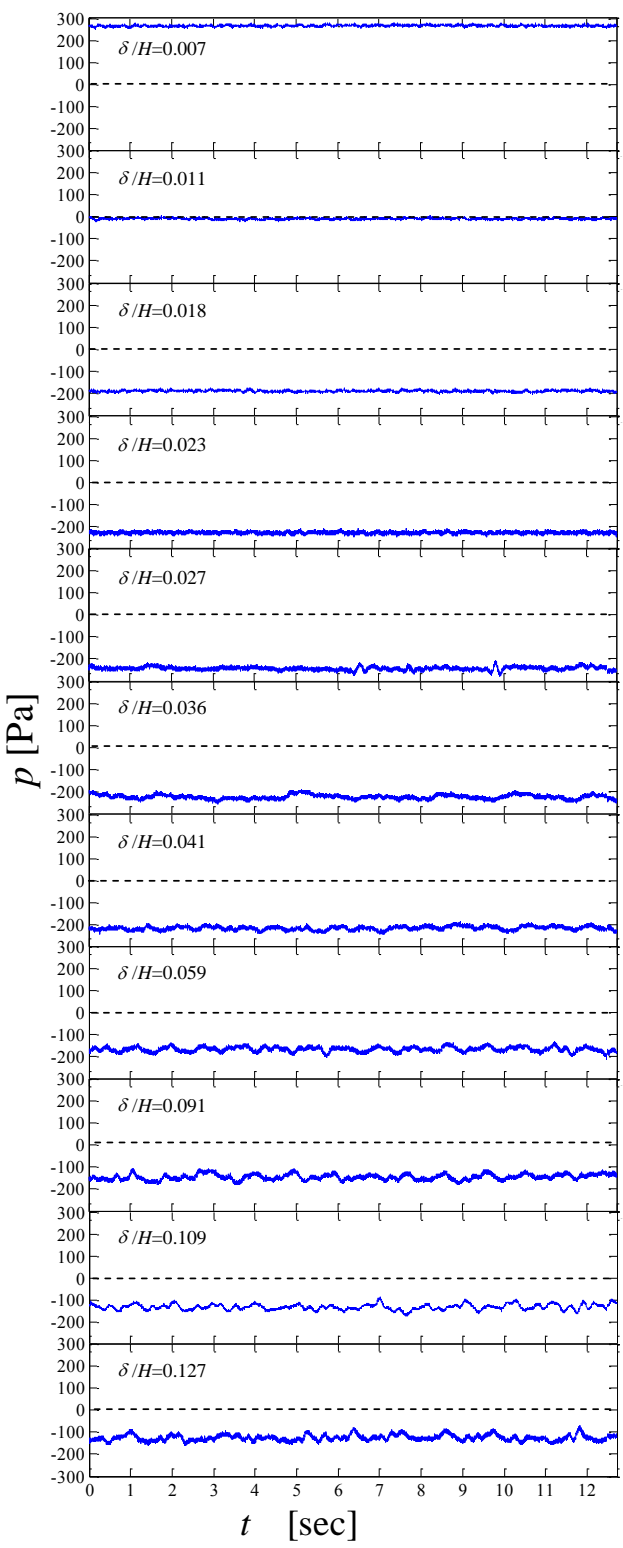

Fig. 6 Pressure fluctuation at various gap height.

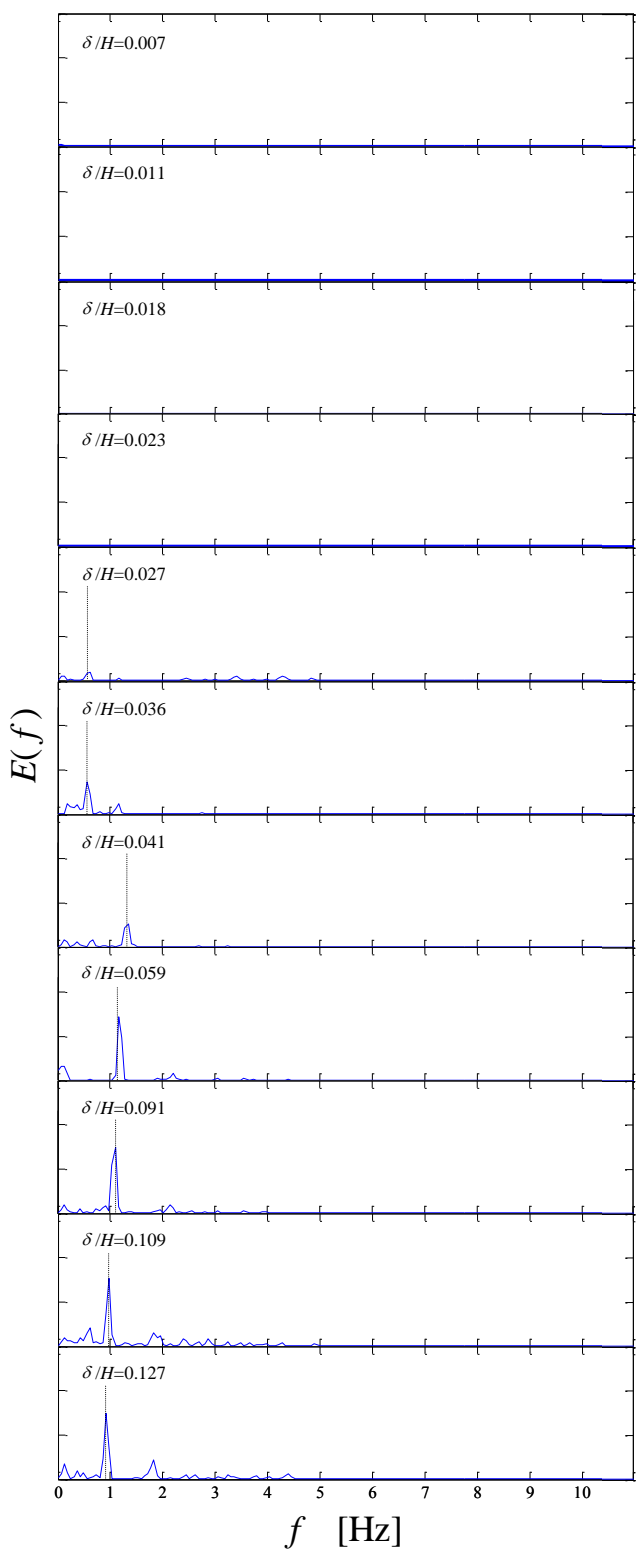

Fig. 7 Spectrum of pressure fluctuation at various gap height. 


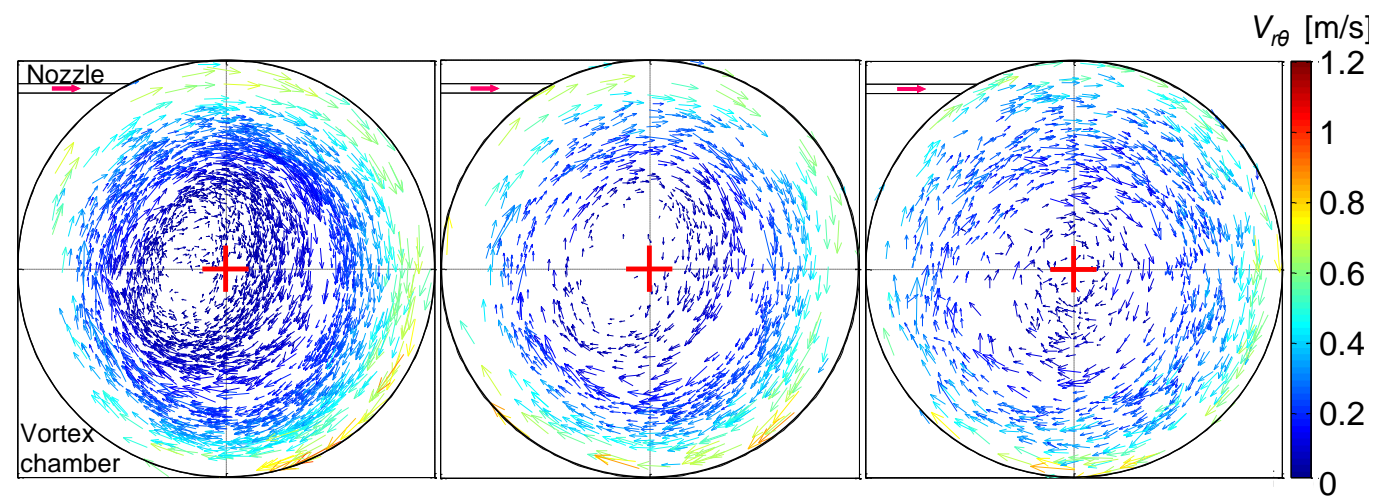

(a) $\delta / H=0.011$

(b) $\delta / H=0.027$

(c) $\delta / H=0.091$

Fig. 8 Velocity distributions at different gap height of $z / H=0.58$

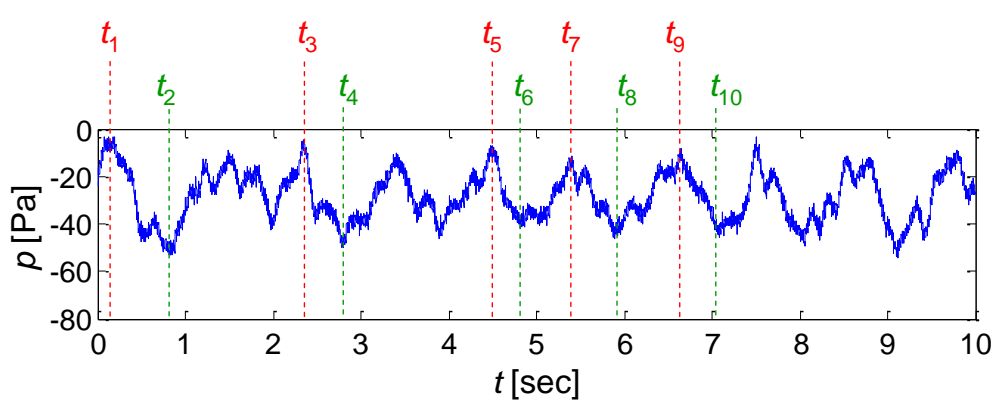

(a) Pressure fluctuation on the wall

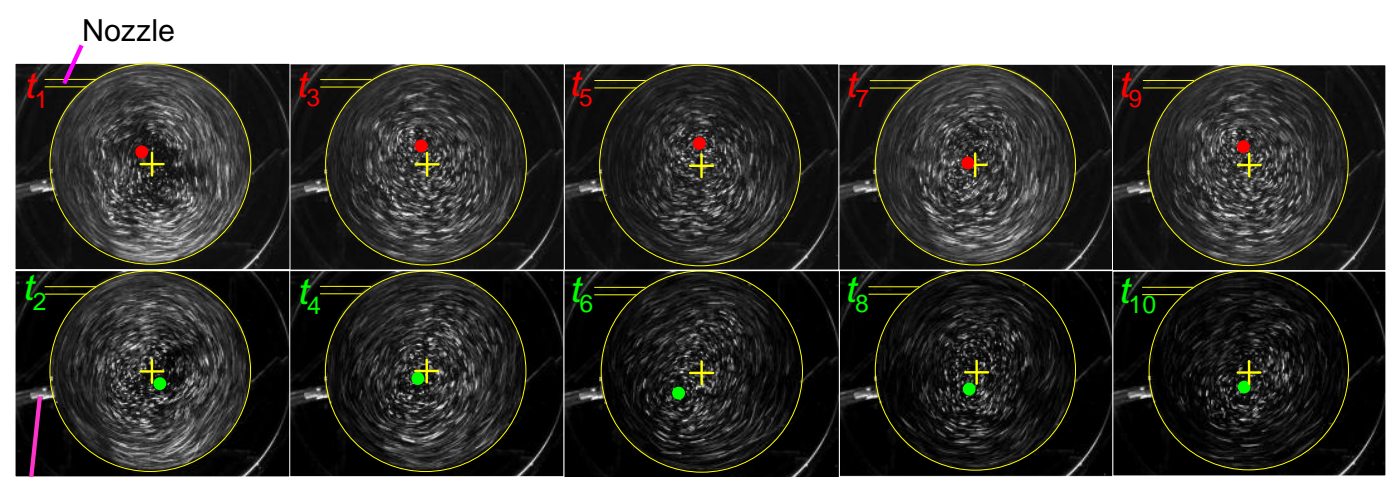

Pressure

transducer

(b) Flow patterns in a vortex cup

Fig. 9 Simultaneous measurement of pressure and swirling flow pattern.

(Measurement height: $z / H=0.58$, Gap height: $\delta / H=0.091$ )

\subsection{Observation of flow field for wide gap height}

Figure 8 illustrates the velocity distributions with PTV (Particle Tracking Velocimetry) at various gap heights. These results were superimposed of 600 results. The height of the illuminating light sheet of halogen lamp was set at $z / H=0.58$. The circle in the figure drawn by solid line shows the inner wall of the cup. The water jet issues from the upper-left placed nozzle shown in each images into the tangential direction, and then a clock-wise swirling flow is generated inside the cup. The velocity component in $r \theta$-plane is defined as $V_{r \theta}$. For all gap heights, the velocity vectors in the outer region of the cup are longer than those in the center region. With $\delta / H=0.011$ or 0.027 , all vectors indicate the tangential direction along the cup's wall, and the center of swirling flow shifts to the left side of the cup. With $\delta / H=0.091$, on the other hand, vectors show in various directions, it is hard to recognize the center of swirling flow. In other words, the stable swirling flow occurs for small gap height, but swirling flow becomes unstable for large gap condition. Thus, swirling flow behavior is attributed to the gap height between the work and the cup. It is seemed 
that the water going through the gap is strongly affected by viscous friction for small gap condition. The swirling flow decays immediately in the gap, and then radial velocity component increases. The gap works as 'damper' to prevent the flow goes freely back and forth between the inside and the outside of the cup. On the contrary, the swirling flow shows perturbative motion under large gap condition. In this case, the swirling flow velocity decays gradually because of little viscous friction in the gap region. It is also visually observed (not shown here) that the flow motion which goes freely back and forth through the gap region is synchronized with the swirling flow perturbation.

Next, the simultaneous measurement of the pressure and the swirling flow was performed to evaluate each direct relationship for wide gap height condition with $\delta / H=0.091$. The result of pressure measurement is shown in Fig. 9(a), and the visualized swirling motion is illustrated in Fig. 9(b). The pressure tap was placed on the inner wall of the cup at $\theta=257^{\circ}$ and $z / H=0.58$ near the back surface of the work piece. The photographs shown here were captured from movies recorded via high speed digital video camera at a frame rate of $200 \mathrm{~Hz}$ for a total time of 10 seconds. The sheet light was illuminated at the same height of the pressure tap at $z / H=0.58$. The each image was extracted at the same time when the pressure reached at maximal or the minimal values. The indexes $t_{1}, t_{2}, t_{3}, \cdots$ on the waveform is corresponded with those on the each image. The occurrence of the periodic pressure perturbation is also recognized on the wall although the mean pressure is higher than that measured at the center of the work piece as shown in Fig. 5. When counting the maximal pressure value, we can recognize the ten peaks for 10 seconds from this pressure waveform. So the dominant frequency is approximately $1 \mathrm{~Hz}$, it is the same value that measured at the center of the work piece as shown in Fig. 7. It is thought that the both pressure perturbations at the work center and on the wall are caused by same phenomenon. Figure 9 (b) shows the swirling flows at the times indicated in Fig. 9 (a) as mentioned above. It can be seen here that the swirling flow rotates periodically at approximately $1 \mathrm{~Hz}$. This frequency is smaller than that of the swirl rotation calculated from the mean swirl velocity of $3.6 \mathrm{~Hz}$. The cause of this difference is still not clear. The red and green colored circular dots drawn in each image indicate the visually-confirmed center of the swirling flow. The upper pictures or lower pictures are captured at the time when the pressure indicated the maximal or minimal values, respectively. In the upper pictures the swirl center shifts to the nozzle side, on the contrary, the center shifts to the opposite side of the nozzle in the lower pictures. These results show that sucking pressure fluctuation in the vortex cup is caused by the periodically eccentric motion of the swirling flow for the wide gap height.

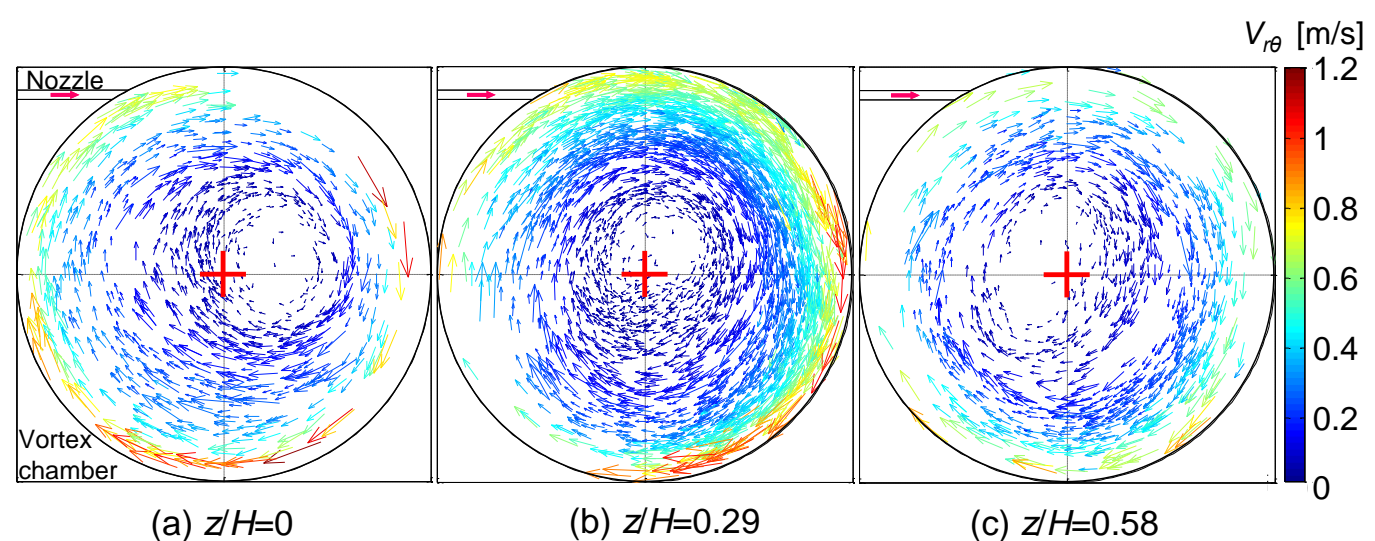

Fig. 10 Velocity distribution at different height of a vortex cup at small gap height for $\delta / H=0.027$.

\subsection{Observation of flow field for small gap height}

The velocity measurement of swirling flow at the different height in the cup is conducted to grasp the detail of swirling behavior in the cup for a small gap height of $\delta / H=0.027$. Figure 10 illustrates the velocity distributions obtained by PTV method. The sheet light was illuminated at the height of $z / H=0,0.29$, and 0.58. All figures show the clockwise rotation of the swirling flow. It is observed that the swirl center is located on the $\theta=90^{\circ}$ side for $z / H=0$, on the cup center for $z / H=0.29$, and on the $\theta=270^{\circ}$ side for $z / H=0.58$. It is thus found that the rotation axis of swirling flow steadily-inclines against the central axis of the vortex cup. Figure 11 presents schematic flow patterns of the jet issuing from the nozzle, which are visualized using the pigment streak-line 
method. The jet issues from the nozzle, and impinges on the inner wall of the cup, and then goes upward along the wall as shown in Fig. 11(a). The downward flow is caused by the jet entrainment of the ambient fluid. These upward and downward flows coexist in the cup, as a result, the swirling flow inclines as illustrated in Fig. 12.

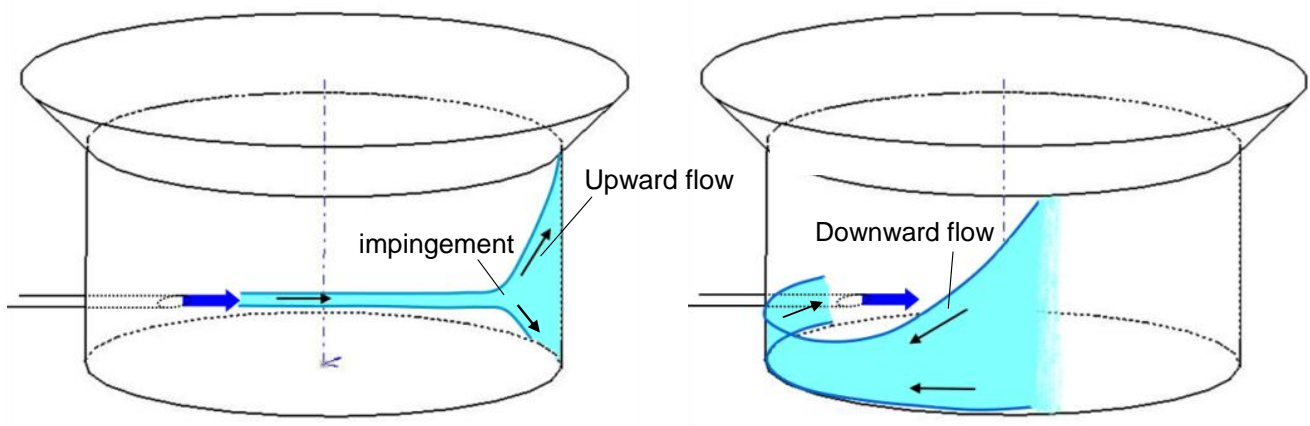

Fig. 11 Outline drawing of flow model caused by a jet.

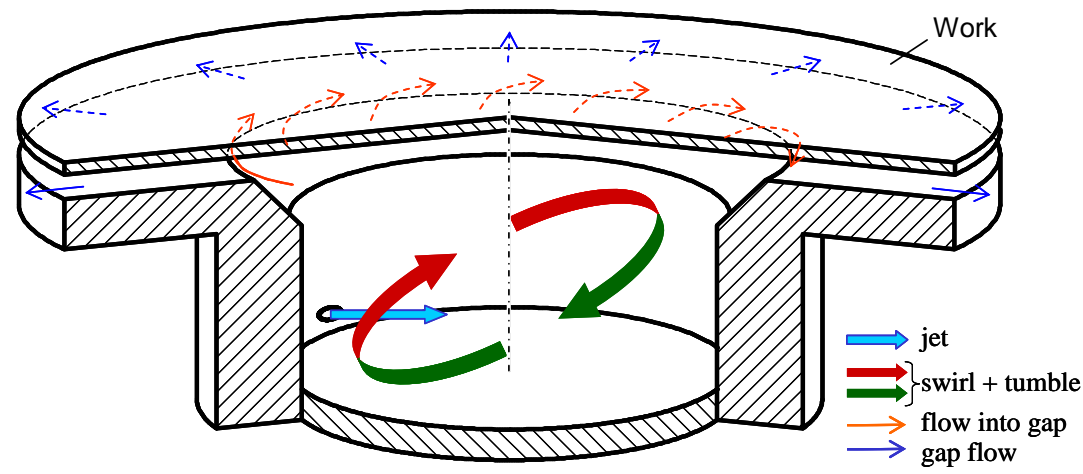

Fig. 12 Schematic view of various flow patterns in the vortex cup for small gap height.

\section{Conclusions}

In conclusion, it should be noted that the results of our experiments showed that the gap height between the vortex cup and the work piece strongly affects on the performance of the vortex levitation approach. In the case of a wide gap height, it is found that the sucking pressure perturbs at the same period with an eccentric motion of the swirling flow, and the perturbation frequency is smaller than that calculated from the swirl velocity. Furthermore, it is proved a strongly relationship between the pressure fluctuation and the swirling motion by the simultaneous measurement of the pressure and the flow field in the cup. In the case of a small gap height, it is confirmed that the sucking pressure has little fluctuation, and that the rotation axis of the swirling flow steadily-inclines against the central axis of the vortex cup. This is due to a jet impingement on the wall and to a jet entrainment of an ambient fluid.

\section{References}

Brandt E.H. (1989), Levitation in physics, Science, 243

Cortes C., Gil A. (2007), Modeling the gas and particle flow inside cyclone separators, Progress in Energy and Combustion Science, 33:409-452

Davis S., Gray J.O., Caldwell D.G. (2008), An end effector based on the Bernoulli principle for handling sliced fruit and vegetables, Robotics and Computer-Integrated Manufacturing, 24(2): 249-257

Dini G., Fantoni G., Failli F. (2009), Grasping leather plies by Bernoulli grippers, CIRP AnnalsManufacturing Technology, 58(1):21-24

Iio S., Umebachi M., Li X., Kagawa T., Ikeda T. (2008), Research on a Non-contact Handling System Using Swirling Flow (Influence of Cup-to-Work Gap Thickness on Swirling Flow Characteristics), SICE Trans. on Industrial Application, 7(12):79-84

Ito S., Ogawa K., Kuroda C. (1979), Decay process of swirling flow in a pipe, Int. Chem. Eng., 19:600-605 
Kitoh O. (1991), Experimental study of turbulent swirling flow in a straight pipe, J. Fluid Mech., 225:445-479

Kumar R., Conover T. (1993), Flow visualization studies of a swirling flow in a cylinder, Experimental Thermal and Fluid Science, 7(3):254-262

Li X., Kawashima K., Kagawa T. (2008), Analysis of vortex levitation, Experimental Thermal and Fluid Science, 32:1448-1454

Li X., Tokunaga H., Cai M., Funaki T., Kawashima K., Kagawa T. (2007), Research on a noncontact handling system using swirling flow (1st Report: Basic characteristics of a vortex chuck), Trans. JFPS, 38(1):1-6

Nishimura K., Takama N., Endoh T., Yoshiki H.(1990), Velocity distribution of swirl flow in a circular cylinder, Manufacture Research, 42(11):653-656

Nissan A.H., Bresan V. P. (1961), Swirling flow in cylinders, AIChE Journal, 7:543-547

Vandaele V., Lambert P., Delchambre A. (2005), Non-contact handling in microassembly (acoustical levitation), Precision Engineering, 29(4):491-505

Waltham C., Bendall S., Kotlicki A. (2003), Bernoulli levitation, Am J Phys, 71(2): 176-179 